\title{
Correction to: Surgical and non-surgical treatment of inguinal hernia during non-elective admissions in the Nationwide Readmissions Database
}

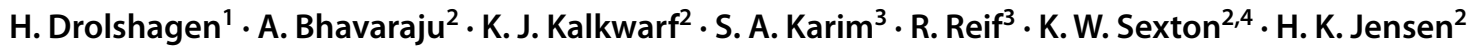

Published online: 27 July 2021

๑) Springer-Verlag France SAS, part of Springer Nature 2021

\section{Correction to: Hernia \\ https://doi.org/10.1007/s10029-021-02441-5}

After online publication, the Authors found that the following data were incorrect.

Abstract, result section: the following sentence " $1.78 \%$ for surgical, $0.49 \%$ for non-surgical, $p<0.01$ "

Should read:

" $0.49 \%$ for surgical, $1.78 \%$ for non-surgical, $p<0.01$ "

Page 3, Results Section, paragraph 2, line 2, the following sentence:

" $1.78 \%$ for surgical, $0.49 \%$ for non-surgical, $p<0.01$ "

Should read:
" $0.49 \%$ for surgical, $1.78 \%$ for non-surgical, $p<0.01$ " The Authors apologise for the oversight. The original article has been corrected.

Publisher's Note Springer Nature remains neutral with regard to jurisdictional claims in published maps and institutional affiliations.

The original article can be found online at https://doi.org/10.1007/ s10029-021-02441-5.

H. K. Jensen

hkjensen@uams.edu

1 College of Medicine, University of Arkansas for Medical Sciences, Little Rock, AR 72205, USA

2 Department of Surgery, Division of Trauma and Acute Care Surgery, University of Arkansas for Medical Sciences, Little Rock, AR 72205, USA

3 Department of Health Policy and Management, Fay W. Boozman College of Public Health, Little Rock, AR 72205, USA

4 Department of Biomedical Informatics, College of Medicine, University of Arkansas, Little Rock, USA 